\title{
Multilayer graphene films grown by Molecular Beam Deposition
}

\author{
Jorge M. Garcia ${ }^{\mathrm{a}, \mathrm{b}, \mathrm{c}}$, Rui He ${ }^{\mathrm{b}}$, Mason P. Jiang ${ }^{\mathrm{b}}$, Jun Yan ${ }^{\mathrm{b}}$, Aron Pinczuk ${ }^{\mathrm{b}}$, Yuri M. Zuev ${ }^{\mathrm{b}}$, Keun Soo Kim ${ }^{\mathrm{b}}$, Philip Kim ${ }^{\mathrm{b}}$, Kirk \\ Baldwin ${ }^{\mathrm{c}, 1}$, Ken W. West ${ }^{\mathrm{c}, 1}$, Loren N. Pfeiffer ${ }^{\mathrm{c}, 1}$
}

${ }^{a}$ MBE Lab, Instituto de Microelectrónica de Madrid, IMM-CNM, CSIC, Spain

${ }^{b}$ Department of Physics and Applied Physics and Applied Mathematics, Columbia University, New York, USA

${ }^{c}$ Bell Labs, Murray Hill, New Jersey, USA

\begin{abstract}
Few-layer graphene films are grown using a molecular beam deposition (MBD) technique in ultra high vacuum by evaporation of atomic carbon and subsequent annealing of the samples at 800-900 ${ }^{\circ} \mathrm{C}$. The graded thickness layers are grown on strip-shaped oxidized silicon substrates which are covered with 300nm thick nickel films deposited by e-beam evaporation. The thickness of the deposited carbon layers changes continuously from $\sim 70 \AA$ to less than $4 \AA$. The relatively narrow optical phonon bands in Raman spectroscopy reveal that good quality multi-layer graphene films form on the Ni surface.
\end{abstract}

Keywords: A Graphene, B. Crystal Growth, E. Inelastic light scattering

\section{Introduction}

Graphene is a single layer of graphite in which carbon atoms are arranged in a honeycomb crystal lattice. The remarkable properties of graphene layers have fueled expectations as a novel material base for applications in future generation devices [1]. The robustness of the quantum properties, exemplified by remarkable observations of quantum Hall effect at room temperature [2], open up a vast potential for new applications such as transistors [3] in which the quantum nature of carrier states could be exploited.

Much of the enormous interest in graphene stems from results obtained in samples mechanically exfoliated from graphite. Practical applications, however, require reliable and well-controlled methods for fabrication of large area graphene films. Graphene layers have been grown by selective sublimation of silicon atoms from a $\mathrm{SiC}$ substrates $[4,5]$. Recently it has been shown that high quality monolayer graphene can be epitaxially grown on $\mathrm{SiC}$ at reduced temperatures by assistance of carbon deposition in Ultra High Vacuum (UHV) [6]. Chemical growth methods have led to the synthesis of graphene from reduction of graphene oxide [7] (RGO) adequate for transparent electrode applications. High quality graphene layers were fabricated using Chemical Vapor Deposition (CVD) on nickel [8$10]$ and copper $[11,12]$ with methane as the source of the carbon atoms. Other ways to synthesize graphitic carbon layers have been successfully obtained by a carbon solid source Molecular Beam Epitaxy (MBE) process on Si(111) [13].

We report the growth of high quality few-layer graphene films by UHV atomic carbon deposition from a hot glassy carbon filament. The growth configuration (see Fig. 1) enables

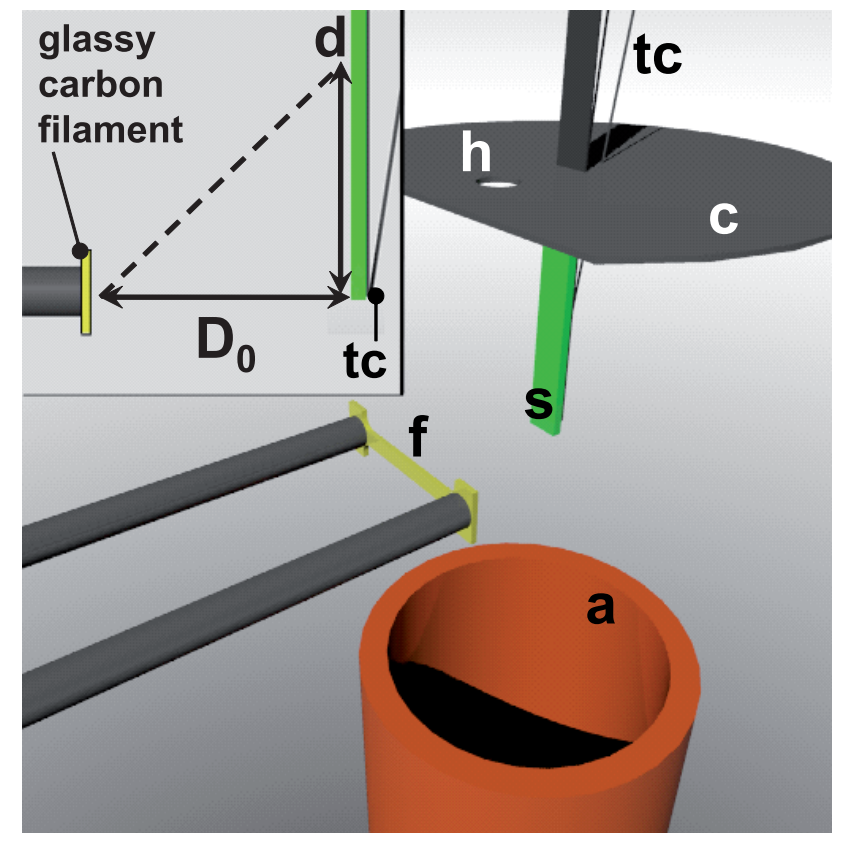

Figure 1: (Color online) Illustration of the experimental set up. The inset depicts a side view with labels of the geometrical parameters $D_{0}=13.3 \mathrm{~mm}$ and $d$.

Email address: jorgem@imm.cnm.csic.es (Jorge M. Garcia)

${ }^{1}$ Present address: Electrical Engineering Dept, Princeton University, Princeton 
the fabrication of graded thickness ultra-thin films onto elongated substrates that support a thin nickel film. After an annealing treatment, graphene layers form on the Ni surface. Raman spectra of the samples show the characteristic optical phonon features that are associated with graphite and graphene. The fabrication of ultra-thin graphitic films by molecular beam deposition (MBD) in UHV creates possibilities for fabrication of large area graphene layers. One of the benefit of this method is the absence of impurities that will allow in situ preparation of ultra high pure metal buffer layers $(\mathrm{Ni}, \mathrm{Cu})$ and high quality graphene layers. Another great benefit of this technique is that it is compatible with in situ vacuum characterization techniques such as scanning tunneling microscope (STM), x-ray photoelectron spectroscopy (XPS), etc.

\section{Materials and methods}

\subsection{Growth set up}

Figure 1 illustrates the MBD growth set up. The UHV chamber has a base pressure of $2 \times 10^{-11}$ Torr. The source for carbon atoms is a vitreous (glassy) carbon filament [14, 15](Fig. 1; $\mathrm{f}$, yellow). This type of cell produces a significantly cleaner carbon film than conventional carbon cells for MBE [16]. The substrate is positioned either in front of the carbon source or it can be lowered in situ into an annealing stage (Fig. 1; a, orange), which is a MBE Knudsen cell with a liner of tantalum that provide a very uniform annealing temperature in a ultra clean environment for samples with many different aspect ratios. During the annealing, the tantalum cap (Fig. 1; c) attached to the sample holder covers the tip of the oven. The temperature is monitored by an optical pyrometer through a small hole (Fig. 1; h) and by a tungsten-rhenium thermocouple (Fig. 1, tc) spot welded to the sample holder. During carbon deposition the chamber pressure rises to $2 \times 10^{-10}$ Torr and the sample is heated to $\sim 350{ }^{\circ} \mathrm{C}$ by the nearby glassy carbon filament which has an operating temperature of $\sim 2100{ }^{\circ} \mathrm{C}$.

\subsection{Raman set up}

Raman spectra are excited at room temperature with an argon-ion laser operating at $\lambda=514.5 \mathrm{~nm}$. The graphene samples are mounted on a xy-z stage for alignment. The z-direction is parallel to the long edges of the sample. The laser spot size is $\sim 20 \mu \mathrm{m}$ and the laser power is kept at $\sim 6 \mathrm{~mW}$. Spectra are recorded by a double grating spectrometer using CCD detection. The spectral resolution is $\sim 5 \mathrm{~cm}^{-1}$. Raman analysis is focussed on measurements of $\mathrm{D}, \mathrm{G}$ and $2 \mathrm{D}$ optical phonons. These peaks are prominent features in the Raman characterization of graphite and graphene [17-20]. The D band is typically associated with defects and grain boundaries [17, 19]. The G band $\left(\sim 1580 \mathrm{~cm}^{-1}\right)$ full width at half maximum (FWHM) is regarded as a measure of disorder and electron-phonon coupling $[17,18]$. The $2 \mathrm{D}$ band $\left(\sim 2700 \mathrm{~cm}^{-1}\right)$ is a zero wave vector two-phonon state $[17,19,20]$.

The substrates are rectangular-shaped $5 \times 40 \mathrm{~mm}^{2}$ silicon with thermally grown $(300 \mathrm{~nm}) \mathrm{SiO}_{2}$ layer on the surface. After the substrates are cleaned with a mixture of sulfuric acid and hydrogen peroxide, a $5 \mathrm{~nm}$ of Ti followed by $300 \mathrm{~nm}$ of $\mathrm{Ni}$ are deposited in a separate e-beam evaporation system.

\subsection{Growth sequence}

A typical growth sequence consists of a cleaning anneal at $800{ }^{\circ} \mathrm{C}$ for $30 \mathrm{~min}$ followed by the deposition of the carbon layer and a final annealing for 30 minutes at 800 to $900{ }^{\circ} \mathrm{C}$. Then the samples are cooled at various controlled rates ranging from $\sim-1$ to $\sim-50{ }^{\circ} \mathrm{C} / \mathrm{sec}$ for the first $200{ }^{\circ} \mathrm{C}$. Annealing at $\gtrsim 1000{ }^{\circ} \mathrm{C}$ after the carbon growth results in the destruction of the nickel film continuity. The conditions that we use are the optimum that we have found so far. Reasonably, the annealing temperatures are slightly lower than the ones employed in CVD due to the big difference in vapor pressures of the $\mathrm{Ni}$ at atmospheric pressure (CVD) and in an UHV environment.

\subsection{Estimation of the gradient on the deposited carbon}

There is a strong gradient in the incoming carbon flux along the long axis of the sample that will be used to explore multiple thicknesses during the same deposition run. We can estimate the deposited carbon thickness before the second annealing $\Theta(d)$, using a Knudsen's cosine law (equation 9 in reference [21])

$$
\Theta(d)=\Theta_{0} /\left(1+\left(d / D_{0}\right)^{2}\right)^{2},
$$

where the parameters $D_{0}$ and $d$ and are defined in the inset of Fig. 1 , and $\Theta_{0}$ is the thickness at normal incidence. The carbon cell is calibrated by measuring the height of a step produced in a not-annealed deposited thick carbon film (13 h deposition) by Atomic Force Microscope (AFM). For that purpose a pattern with trenches is defined by UV photo-lithography and oxygen dry plasma etch ( $5 \mathrm{~min}$, sccm $30,100 \mathrm{~V})$. The carbon growth rate is $0.123 \AA / \mathrm{min}$ for $14.25 \mathrm{~A}$ of DC current through the glassy carbon filament.

Our MBD method can be used to control the amount of deposited carbon with sub-monolayer precision. It is possible to easily change either the deposition time or the growth rate. Our equipment has been designed for the growth of graded elongated samples or small $3 \times 3 \mathrm{~mm}^{2}$ graphene films of uniform thickness.

\section{Results and discussion}

Figure 2(a) shows the Raman spectra of a carbon film on Nickel without any annealing treatment. There are no traces of D, G, or 2D Raman resonances. Panels (b-f) show the evolution of the spectra as the deposited distance $d$ to the edge of the sample increases (i.e. as the carbon thickness decrease) in a sample that has been annealed at $900{ }^{\circ} \mathrm{C}$ for $30 \mathrm{~min}$ and cooled down at $-10^{\circ} \mathrm{C} / \mathrm{sec}$. The presence of well-defined $\mathrm{G}$ and $2 \mathrm{D}$ (and D) features reveals the formation of graphitic layers. The spectra in Fig. 2 have been normalized to the G peak intensity, and have been fit to a Gaussian lineshape in the cases of D band. A Lorentzian lineshape was used for the $G$ band [18].

The data in Fig. 2 is plotted as a function of the distance $d$, instead of carbon coverage, to avoid confusion between the 


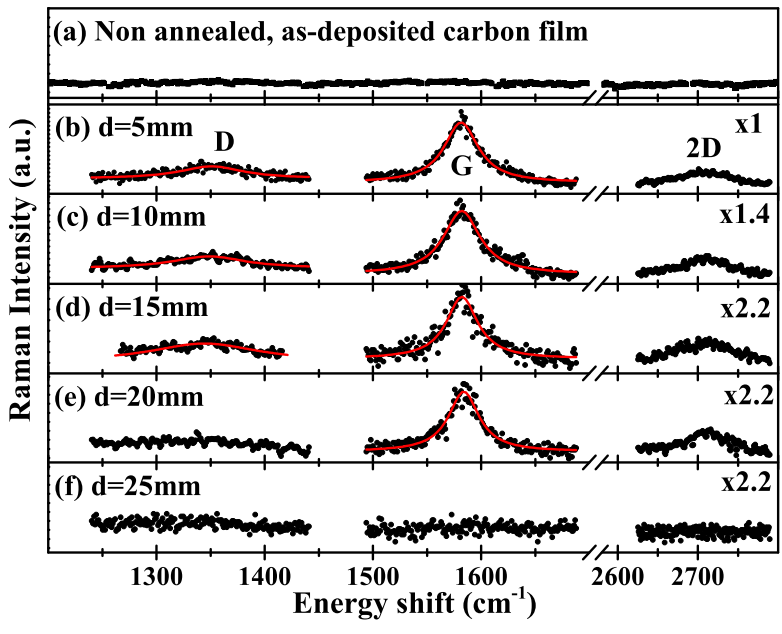

Figure 2: (Color online) Raman spectra of ultra-thin carbon layers grown on a strip-shaped $5 \times 30 \mathrm{~mm}^{2}$ substrate. (a) As-deposited carbon before annealing. (b-f) Evolution of the D, G and $2 \mathrm{D}$ resonances with distance $d$. For regions with $d \sim 25 \mathrm{~mm}$ or larger (f) there are no characteristic resonances of graphene.

thickness of deposited carbon $\Theta$ and the actual precipitated carbon on the surface after the second annealing. The estimated thickness $\Theta$ corresponding to the labels in the panels of Fig. 2 are (according to equation 1): (b) $56.8 \AA, d=5 \mathrm{~mm}$; (c) $30.2 \AA$, $d=10 \mathrm{~mm}$; (d) $14.3 \AA, d=15 \mathrm{~mm}$; (e) $7.0 \AA, d=20 \mathrm{~mm}$; and (f) $3.6 \AA, d=25 \mathrm{~mm}$. The presence of the Raman resonances is a result of the formation of graphite. For the thinnest regions of deposited carbon (just before the Raman resonances disappear) these results suggest the formation of few graphite layers, that is to say, few multilayers of graphene.

The D band has a width between $70-92 \mathrm{~cm}^{-1}$, revealing some degree of disorder associated with a broad distribution of crystalline sizes as confirmed by AFM (inset of Fig. 3) which shows many grains with sizes $\lesssim 1 \mu \mathrm{m}$. The surface, independent of the position $d$ of AFM measurement, has the same grain structure with a root-mean-square (RMS) roughness of about $120 \AA$. Therefore, this multigrain structure of the multilayer graphene film is mainly attributed to the morphology of the underlying Ni film.

Figure 3 presents the evolution of the $G$ band width and the relative height intensities $\mathrm{I}(2 \mathrm{D}) / \mathrm{I}(\mathrm{G})$ and $\mathrm{I}(\mathrm{D}) / \mathrm{I}(\mathrm{G})$ with distance $d$. As $d$ increases and the amount of deposited carbon decreases, the $\mathrm{G}$ band narrows from $38 \mathrm{~cm}^{-1}$ to $34 \mathrm{~cm}^{-1}$. The Raman signatures disappear for $d \sim 25 \mathrm{~mm}$ or larger, i.e. for an estimated thickness equivalent to less than $3.6 \AA$; suggesting that only a small fraction of the deposited carbon does not segregate onto the Ni surface upon cooling as observed before [10].

The relative intensity $\mathrm{I}(2 \mathrm{D}) / \mathrm{I}(\mathrm{G})$ shown in Fig. 3 slightly increases when the carbon thickness decreases. We have much less intense 2D peaks to the ones obtained in CVD works but still $>3$ times better than the ones obtained by RGO (supplementary information [7]). It is conceivable that this source of broadening could be the impact of grain size and breakdown of wave vector conservation in Raman scattering. The thinnest re-

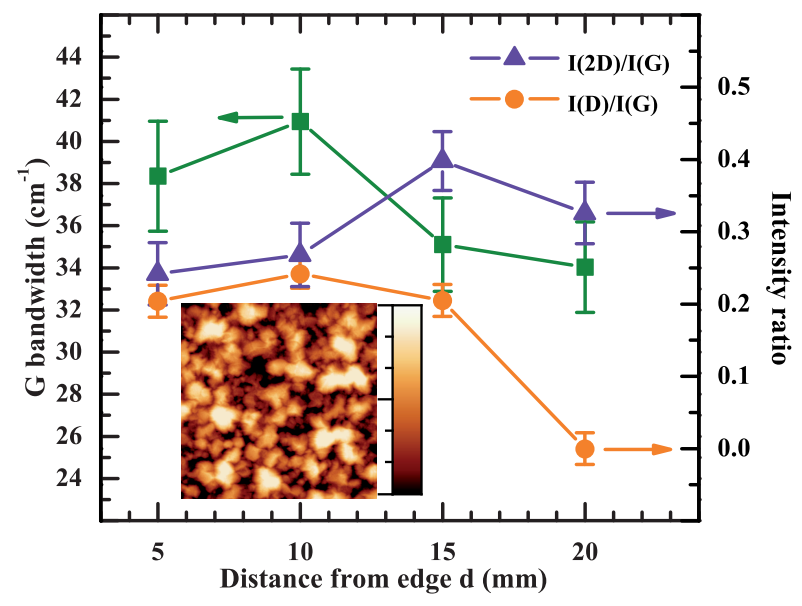

Figure 3: (Color online) Evolution with distance $d$ of $\mathrm{G}$ bandwidth and relative height intensities $\mathrm{I}(2 \mathrm{D}) / \mathrm{I}(\mathrm{G})$ and $\mathrm{I}(\mathrm{D}) / \mathrm{I}(\mathrm{G})$. Inset: $8 \times 8 \mu \mathrm{m}^{2}$ AFM image of a sample with $74 \AA$ deposited carbon. AFM color palette scale is $1100 \AA$.

gions present very low $\mathrm{I}(\mathrm{D}) / \mathrm{I}(\mathrm{G})$ values suggesting that as the quality of these layer is high. The quality of the layers clearly improves for $d>15 \mathrm{~mm}$, corresponding to $\sim 14 \AA$ of deposited carbon prior to the second annealing process. We find that the best graphene layers are obtained with $7 \AA$ of carbon. The limits on the obtained quality seems to be the surface morphology of the nickel buffer layer. Further work is in progress to improve the quality of the Ni buffer layer.

Multilayer graphene films produced by CVD on Ni [9] and $\mathrm{Cu}$ [11] display a $\mathrm{G}$ band FWHM of $\sim 26 \mathrm{~cm}^{-1}$ and $\sim 22 \mathrm{~cm}^{-1}$, better than that in our thinnest film. On the other hand, multilayer graphene grown by RGO [7] shows a significantly larger $\mathrm{G}$ band width of about $55 \mathrm{~cm}^{-1}$. This $\mathrm{G}$ band width analysis indicates that the quality of layers grown by MBD is closer to those of CVD grown graphene films. The D peak intensities in the thinner regions are much lower to those reported for RGO graphene grown samples [7] but somehow similar than those reported for CVD grown films measured with microRaman scattering in regions of graphene grains away from their edges $[8,9]$. Other methods have succeeded in using a solid source of carbon for MBE growth of graphitic carbon films [13] on $\mathrm{Si}(111)$ showing clear $s p^{2}$ character in the XPS spectra. In comparison, our samples present higher quality with a much narrower $\mathrm{G}$ band and less intense $\mathrm{D}$ peak.

\section{Conclusions}

To conclude, a method for the fabrication graphene layers has been developed by using MBD on Ni employing a highly efficient carbon source. The amount of deposited atomic carbon can be accurately controlled. Raman spectroscopy characterization indicates that the MBD samples consist of good quality few layer graphite/graphene.

\section{Acknowledgments}

This work is supported by ONR (N000140610138 and Graphene Muri), NSF (CHE-0117752 and CHE-0641523), 
NYSTAR, Spanish CAM (Q\&C Light, S2009ESP-1503), Spanish MICINN (NANINPHO-QD, TEC2008-06756-C0301, CONSOLIDER QOIT and Salvador de Madariaga Grant no. PR2007-0036).

\section{References}

[1] A. K. Geim and K. S. Novoselov, Nature Materials 6 (2007) 183.

[2] K. S. Novoselov, Z. Jiang, Y. Zhang, S. V. Morozov, H. L. Stormer, U. Zeitler, J. C. Maan, G. S. Boebinger, P. Kim, and A. K. Geim, Science 315 (2007) 1379.

[3] Yu-Ming Lin, Keith A. Jenkins, Alberto Valdes-Garcia, Joshua P. Small, Damon B. Farmer and Phaedon Avouris, Nano Lett. 9 (2009) 422.

[4] C. Berger, Z. Song, X. Li, X. Wu, N. Brown, C. Naud, D. Mayou, T. Li, J. Hass, A. N. Marchenkov, E. H. Conrad, P. N. First, and W. A. de Heer, Science 312 (2006) 1191.

[5] K. V. Emtsev, A. Bostwick, K. Horn, J. Jobst, G.L. Kellogg, L.Ley, J.L. McChesney, T. Ohta, S.A. Reshanov, J.Rhrl, E.Rotenberg, A.K. Schmid, D.Waldmann, H.B. Weber and T.Seyller, Nature Matt. 8 (2009) 203.

[6] A. Al-Temimy, C. Riedl, and U. Starke, Appl. Phys. Lett. 231907 (2009) 95.

[7] G. Eda, G. Fanchini, and M. Chhowalla, Nature Nanotechnol. 3 (2008) 270.

[8] K. S. Kim, Y. Zhao, H. Jang, S. Y. Lee, J. M. Kim, K. S. Kim, J.-H. Ahn, P. Kim, J.-Y. Choi, and B. H. Hong, Nature 457 (2009) 706.

[9] A. Reina, X. Jia, J. Ho, D. Nezich, H. Son, V. Bulovic, M. S. Dresselhaus, and J. Kong, Nano Lett. 9 (2009) 30.

[10] Q. Yu, J. Lian, S. Siriponglert, H. Li, Y. P. Chen, and S.-S. Pei, Appl. Phys. Lett. 93 (2008) 113103.

[11] X. Li, W. Cai, J. An, S. Kim, J. Nah, D. Yang, R. Piner, A. Velamakanni, I. Jung, E. Tutuc, S. K. Banerjee, L. Colombo, and R. S. Ruoff, Science 324 (2009) 1312.

[12] W. Cai, Y. Zhu, X. Li, R. D. Piner, and R. S. Ruoff, Appl. Phys. Lett. 95 (2009) 123115.

[13] J. Hackley, D. Ali, J. DiPasquale, J. D. Demaree, and C. J. K. Richardson, Appl. Phys. Lett. 95 (2009) 133114.

[14] L. N. Pfeiffer, R. de Picciotto, K. W. West, K. W. Baldwin, and C. H. L. Quay, Appl. Phys. Lett. 87 (2005) 073111.

[15] L.N. Pfeiffer and K. W. West, US Patent 7329595.

[16] Sources for carbon doping, Dr. Eberl MBE-Komponenten.

[17] A. C. Ferrari,and J. Robertson, Phys. Rev. B 61 (2000) 14095.

[18] J. Yan, Y-B Zhang, P. Kim and A. Pinczuk, Phys. Rev. Lett. 98 (2007) 166802.

[19] C. Casiraghi, A. Hartschuh, H. Qian, S. Piscanec, C. Georgi, A. Fasoli, K. S. Novoselov, D. M. Basko, and A. C. Ferrari, Nano Lett. 9 (2009) 1433.

[20] A. Gupta, G. Chen, P. Joshi, S. Tadigadapa, and P.C. Eklund, Nano Lett. 6 (2006) 2667.

[21] L. Holland, and W. Steckelmacher, Vacuum 11 (1952) 346. 